\title{
Dosimétrie environnementale des patients à la suite de leur examen d'imagerie médicale
}

\author{
J. LEMOINE ${ }^{1}$, J.C. BOURRE ${ }^{2}$, J.Y. GIRAUD ${ }^{3}$
}

(Manuscrit reçu le 23 juin 2011, accepté le 2 octobre 2011)

RÉSUMÉ Les patients sortant du service de médecine nucléaire émettent du rayonnement ionisant, il est donc important de savoir si la dose que reçoit l'entourage direct du patient est dangereuse, en particulier pour les personnes à risque comme les femmes enceintes et les enfants jeunes. Une partie des patients sortent du service juste après leur image, en conséquence, la mesure de leur radioactivité a été faite après leur sortie de la salle d'imagerie. Je me suis employé à mesurer ces patients afin de pouvoir quantifier cette dose. J'ai utilisé un radiamètre mesurant en continu la radioactivité, cela m'a permis de voir la différence nette entre un patient radioactif ou non. Toutes les informations utiles pour mon article étaient répertoriées dans la base de données du service. J'ai donc utilisé ces données pour étudier ma population et donner du sens aux doses mesurées. Les temps entre l'injection du produit et la mesure, variables d'un examen à l'autre et la différence de période suivant l'élément radioactif utilisé, ont compliqué la comparaison des doses mesurées. J'ai donc résonné de façon absolue sur les doses et très peu relativement les unes par rapport aux autres. Les mesures ont montré qu'aucun patient sortant du service après une image ne sortait en dépassant la limite du service qui est de $20 \mu \mathrm{Sv} / \mathrm{h}$ et ne pouvait donner à son entourage une dose atteignant le $\mathrm{mSv}$, dose maximale pour le public par an. J'ai également mesuré des patients recevant un traitement à l'iode 131. Il est arrivé qu'un patient dépasse la limite du service mais la dose que l'entourage reçoit reste inférieure au mSv.

ABSTRACT Radiation doses in the surrounding of patients undergoing a nuclear medecine exam.

As patients leaving the department of nuclear medicine emit ionizing radiation, it is important to know if the dose received by the patient's entourage is dangerous, especially for those at risk such as pregnant women and young children. Some patients left the service immediately after their imaging; therefore, measurement of their radioactivity was carried out after they left the imaging room. I applied myself to measuring these patients in order to quantify this dose. I used a radiameter which continuously measured the radioactivity, which allowed me to see the difference between whether a patient was radioactive or not. All the relevant information for my article was listed in the database service. Therefore, I used these data to study the population and give meaning to the measured doses. The time between the injection and measurement varies from one examination to another, and the difference in time after the radioactive element used complicated the comparison of measured doses. So I mainly considered the doses in an absolute way rather than comparing them with each other. The measurements showed that no patient who left the service after imaging ever exceeded the limit of the service, which is $20 \mu \mathrm{Sv} / \mathrm{h}$, and could not give his entourage a dose reaching the $\mathrm{mSv}$, the maximum dose for the public each year.

Faculté de Médecine de Grenoble.

Médecin nucléaire au Centre hospitalier universitaire (CHU) de Grenoble.

Physicien médical au CHU de Grenoble. 
I also measured the patients treated with iodine-131. It happened that a patient exceeded the limit of the service but the dose that the entourage received was below the limit of $1 \mathrm{mSv}$.

Keywords: Radiation dose / nuclear medecine

\section{Introduction}

Au sein du CHU de Grenoble, le service de médecine nucléaire utilise un type particulier d'imagerie. Elle est réalisée grâce au rayonnement ionisant produit par le patient après injection de produit radioactif. Les images obtenues servent ensuite à aider le médecin à prendre une décision plus avisée vis-à-vis de la situation du patient. Suivant l'isotope radioactif utilisé avec un traceur ou non, les images montrent la présence ou l'absence de tout type de problème. Néanmoins ces examens sont contraignants à cause de la radioactivité, pour le patient, son entourage et les manipulateurs. Du point de vue de la radioprotection du patient, il peut être intéressant de voir si ce dernier peut devenir une source d'irradiation plus ou moins intense pour les personnes qu'il sera amené à croiser en sortant du service. Il existe plusieurs éléments radioactifs utilisés en médecine nucléaire dans plusieurs examens différents. Ici nous ne parlerons que des 3 isotopes principaux le Fluor, le Technétium et le Thallium. Ces éléments sont associés à un traceur qui va fixer les parties du corps des patients que l'on veut voir. Cette combinaison d'éléments est appelée radiotraceur. Les examens dans lesquels ils sont utilisés et après lesquels nous avons fait nos mesures sont la Tomographie par émission de positions (TEP), la scintigraphie osseuse, la fraction d'éjection du ventricule gauche (FEVG) et la scintigraphie cardiaque. L'activité injectée au patient dépend de l'examen. Le patient irradie donc plus ou moins son entourage selon l'examen qu'il a passé. Si ce phénomène est contrôlé et connu à l'intérieur du service, il n'en est pas de même pour les personnes rencontrées à l'extérieur. Le service de Médecine nucléaire n'est pas seulement destiné à l'imagerie. Des traitements à l'iode sont aussi délivrés pour les personnes atteintes d'hyperthyroïdie. Ces patients sortent du service juste après ingestion d'une pastille d'iode radioactive et il nous a donc paru pertinent de les mesurer avant leur sortie du service.

L'objectif principal de cette étude est de comparer les débits de dose émis par les patients à la sortie de leur examen au débit de dose limite de $20 \mu \mathrm{Sv} / \mathrm{h}$ établi dans le service de médecine nucléaire. L'objectif secondaire est d'évaluer l'enveloppe de risque de l'entourage du patient au regard de la limite de $1 \mathrm{mSv} / \mathrm{an}$ prévu pour le public par la législation.

\section{Matériel et méthodes}

Les mesures ont été faites sur des patients venant de réaliser les examens d'imagerie et le traitement à l'iode. Tous les patients sortants ont été mesurés sans 
critères d'exclusion. Les mesures ont été faites entre le 13 avril et le 18 mai 2011 dans le service de Médecine nucléaire du CHU de Grenoble. Le nombre de patients étant limité par l'afflux dans le service, la limite de 30 patients pour avoir des systèmes paramétriques lors des tests statistiques n'a pas pu être respectée pour certains examens durant la période de mesure. L'étude a été réalisée conformément aux bonnes pratiques de la médecine, dans le respect des règles éthiques, et conformément à la législation relative à l'informatique et libertés.

\subsection{Description du matériel utilisé}

Dans un premier temps, nous avons testé plusieurs matériels de détection comme la BABYLINE 61, un radiamètre portatif et un détecteur à scintillation.

Cependant, la BABYLINE 61 et le spectromètre ont été utilisés dans un but pédagogique pour se familiariser avec une partie des outils pouvant servir à mesurer la radioactivité des patients. Les mesures faites avec ce type de matériel ont été faites avant que l'étude ne commence. Il a été décidé de ne pratiquer les mesures sur patient qu'avec le radiamètre portatif. Le radiamètre portatif allant de $10 \mathrm{nSv}$ à $100 \mathrm{mSv}$ pour une gamme d'énergie de $30 \mathrm{KeV}$ à $4,4 \mathrm{MeV}$. Il s'agit d'un radiamètre de ESM le FH 40G-L10. Il mesure le débit de dose équivalent photons, donc la dose efficace que nous cherchons. Il a été utilisé en mode normal, où il suffisait d'attendre que la valeur se stabilise pour pouvoir lire le débit de dose correct.

Enfin, le bruit de fond étant plus de 10 fois inférieur aux valeurs mesurées, il a été considéré comme négligeable.

\subsection{Protocole de mesure et d'acquisition des données patients après leur examen}

Tous les patients, bénéficiant un jour donné de l'un des examens, étaient mesurés. Ils sortaient de la salle d'examen et ils étaient mesurés debout ou allongé pour les patients alités. Les mesures étaient faites à 1 mètre et à 0,5 mètre, la distance gardée strictement identique grâce à un mètre étalon. Pour les examens de scintigraphie osseuse dynamique, fraction d'éjection et pour la première injection de la scintigraphie cardiaque, les mesures ont été faites, idéalement, avant que les patients n'aient pu éliminer biologiquement la radioactivité. En ce qui concerne les autres mesures, la quasi-totalité des patients étaient déjà passés aux toilettes. Les patients ont été prévenus oralement de la mesure post-examen.

Nous notions l'heure de mesure afin de pouvoir déterminer combien de temps après l'injection, la mesure a été faite. Nous avons ensuite recueilli l'heure à 
laquelle l'examen a eu lieu, l'activité injectée en Becquerel, le poids du patient en kilogramme, l'âge et le sexe. L'activité indiquée tenait déjà compte de la décroissance radioactive ayant eu lieu entre le moment de fabrication et le moment de l'injection. De ces données, nous avons calculé la dose émise en moyenne à la sortie de l'hôpital ou du service et donc nous avons pu voir si leur émission de rayonnement est dangereuse pour le public entourant le patient.

Nous avons utilisé le logiciel Statview pour la statistique, les corrélations, moyennes, écarts-type, ainsi que le test de Student pour séries appariées paramétriques dans le cas des scintigraphies myocardiques et de la TEP et un test de Wilcoxon pour les comparaisons non paramétriques.

\subsection{Méthode d'analyse de l'objectif secondaire}

L'objectif secondaire de l'étude était de vérifier le public que croisera le patient ainsi que la famille qui sera à ses côtés, sans pour autant être considérés comme des personnes aidantes. Nous avons utilisé les périodes physiques et effectives des éléments radioactifs pour exploiter les mesures de débits de dose.

Pour rappel, la radioactivité naturelle en France est de 3,3 mSv/an, soit $377 \mathrm{nSv} / \mathrm{h}$. Cependant, le bruit de fond mesuré dans le service n'était que de $100 \mathrm{nSv} / \mathrm{h}$, nous avons pris cette dernière valeur car étant inférieure, elle garantit une majoration du résultat. À titre indicatif, le temps d'exposition du public au rayonnement par le patient a été évalué à $10 \mathrm{~min}$, cela correspond à un trajet en transport en commun ou à un passage dans une file d'attente.

La période effective du ${ }^{99} \mathrm{~m}$ Tc a été obtenue grâce aux patients ayant eu au cours de leur scintigraphie osseuse, une injection et deux mesures. Nous avons effectué une régression des 9 exponentielles correspondant aux 9 patients. Dégageant une période moyenne avec le logiciel Regressi, celle-ci est l'inverse de la constante radioactive correspondant à la tangente de la pente à l'origine. Le point correspondant au temps nul est le débit de dose à l'origine. Cela nous a permis de dégager une période effective moyenne et un débit de dose à l'origine moyen à 1 mètre et à 0,5 mètre. Grâce à cela et aux formules précédemment citées, nous avons pu en déduire les valeurs du débit de dose total, de la durée d'émission, et de la dose reçue en 10 minutes.

Pour l'iode, une période effective a pu être trouvée sur le site de l'Institut de la radioprotection et de la sureté nucléaire, elle est établie à 7 jours. Nous pourrons donc comparer les résultats obtenus avec la période effective avec ceux de la période physique.

Pour les examens où nous avons une seule mesure, nous nous sommes servis de la période physique car il est impossible de retrouver la courbe de décroissance 
avec un seul point. Il est important de noter que la période physique est toujours plus longue que la période effective. Les calculs faits seront donc une majoration du risque encouru par les personnes amenées à le croiser. De ces doses nous pourrons voir si le public ou la famille qui rend visite au patient chez lui reçoit une dose supérieure ou égale à $1 \mathrm{mSv}$.

\section{Résultats}

La population totale mesurée au cours de la période de mesure est de 145 patients, 70 femmes et 75 hommes. L'âge moyen était de 69,8 ans ( $+/-8,9$ ans) et le poids moyen de $56,9 \mathrm{~kg}(+/-7,0 \mathrm{~kg})$. Le tableau I détaille les caractéristiques de cette population en la classant par type d'examen effectué. Les corrélations faites sur les mesures obtenues n'ont pas montré que la dose efficace reçue était proportionnelle à l'inverse de la distance au carré, alors que c'est normalement le cas. Sauf indication, toutes les mesures faites à 1 mètre sont significativement différentes de celles faites à 0,5 mètre. Après vérification dans Statview, toutes les données suivent une loi normale.

Les activités injectées varient suivant l'isotope radioactif utilisé et sont généralement adaptées aux poids des patients. Ainsi pour les examens d'imagerie au Technétium, les données montrent que les activités injectées sont beaucoup plus importantes que celles de Fluor ou de Thallium. Sachant que celui-ci a une période courte, la dose efficace diminue rapidement après l'examen.

L'activité injectée de l'iode est en moyenne plus importante car elle est utilisée en radiothérapie interne. Il est donc normal de retrouver des valeurs importantes.

Une illustration de ceci est que pour des doses semblables à 1 mètre après une TEP au FDG et une fraction d'éjection au Sestamibi marqué au ${ }^{99 \mathrm{~m}} \mathrm{Tc}$, l'activité injectée en Fluor est très inférieure à celle injectée en Technétium. Cette égalité étant observée à des temps différents montre que le Fluor irradie autant 2 heures après l'injection que le Technétium $20 \mathrm{~min}$ après injection, malgré la grande différence de d'activité.

\subsection{Résultats pour la scintigraphie osseuse}

La moyenne d'activité injectée aux patients en scintigraphie osseuse est inférieure a $550 \mathrm{MBq}$ car l'étude inclut deux enfants de 7 et 11 ans qui ont reçu une activité de 205 et $318 \mathrm{MBq}$, ces valeurs font chuter la moyenne de l'échantillon qui serait de 568,6 MBq (+/- 58,6 MBq). Il existe une activité injectée minimum pour les adultes de $550 \mathrm{MBq}$ pour tout patient présentant un poids inférieur à $74 \mathrm{~kg}$ mais ce seuil est plus bas pour les enfants et l'étude en a inclu. La présence de ce minimum 
TABLEAU I

Caractéristiques de population.

Population specifications.

\begin{tabular}{|c|c|c|c|c|c|c|}
\hline TEP & & & & & & \\
\hline \multirow[t]{2}{*}{ Nombre de patients } & Femme & Homme & Total & & & \\
\hline & 19 & 34 & 53 & & & \\
\hline Poids moyen (kg) & & & $72,5(+/-17,8)$ & & & \\
\hline Extremum & & & $35<->125$ & & & \\
\hline Age moyen (ans) & & & $61,3(+/-14,2)$ & & & \\
\hline Extremum & & & $12<->86$ & & & \\
\hline Scintigraphie osseuse & & & Précoce & & & Tardive \\
\hline \multirow{2}{*}{ Nombre de patients } & Femme & Homme & Total & $\mathrm{F}$ & $\mathrm{H}$ & Total \\
\hline & 8 & 5 & 13 & 24 & 19 & 43 \\
\hline Poids moyen (kg) & & & $60,2(+/-18,4)$ & & & $68,4(+/-17,2)$ \\
\hline Extremum & & & $34<->80$ & & & $22<->98$ \\
\hline Age moyen (ans) & & & $47,5(+/-24,7)$ & & & $61,8(+/-20,2)$ \\
\hline Extremum & & & $7<->89$ & & & $7<->90$ \\
\hline \multicolumn{7}{|c|}{ Fraction d'éjection du ventricule gauche } \\
\hline \multirow[t]{2}{*}{ Nombre de patients } & Femme & Homme & Total & & & \\
\hline & 6 & 2 & 8 & & & \\
\hline Poids moyen (kg) & & & $62,1(+/-12,5)$ & & & \\
\hline Extremum & & & $47<->80$ & & & \\
\hline Age moyen (ans) & & & $49,2(+/-11,4)$ & & & \\
\hline Extremum & & & $36<->71$ & & & \\
\hline \multicolumn{7}{|c|}{ Scintigraphie cardiaque (Thallium) } \\
\hline \multirow[t]{2}{*}{ Nombre de patients } & Femme & Homme & Total & & & \\
\hline & 22 & & 30 & & & \\
\hline Poids moyen (kg) & & & $77,6(+/-19,3)$ & & & \\
\hline Extremum & & & $32<->137$ & & & \\
\hline Age moyen (ans) & & & $63,2(+/-14,0)$ & & & \\
\hline Extremum & & & $33<->86$ & & & \\
\hline \multicolumn{7}{|c|}{ Scintigraphie cardiaque (Technétium) } \\
\hline \multirow[t]{2}{*}{ Nombre de patients } & & Homme & Total & & & \\
\hline & & 3 & 3 & & & \\
\hline Poids moyen (kg) & & & $84,3(+/-10,8)$ & & & \\
\hline Extremum & & & $72<->92$ & & & \\
\hline Age moyen (ans) & & & $63,3(+/-9,2)$ & & & \\
\hline Extremum & & & $54<->74$ & & & \\
\hline \multicolumn{7}{|c|}{ Résultats pour l'ingestion d'Iode 131} \\
\hline \multirow[t]{2}{*}{ Nombre de patients } & Femme & Homme & Total & & & \\
\hline & 5 & 3 & 8 & & & \\
\hline Poids moyen (kg) & & & $63,6(+/-7,6)$ & & & \\
\hline Extremum & & & $58<->80$ & & & \\
\hline Age moyen (ans) & & & $51,9(+/-19,3)$ & & & \\
\hline Extremum & & & $25<->84$ & & & \\
\hline
\end{tabular}


et le poids moyen de l'échantillon présent ne permettent pas de montrer l'existence de la formule de proportionnalité entre le poids et la dose utilisée en médecine nucléaire.

Pour la scintigraphie osseuse précoce, les mesures ont été faites 20 minutes après injection. Ces patients, une fois leur examen terminé peuvent être en contact avec d'autres personnes à l'hôpital ou à domicile. Nous les avons obtenus sur une population de 13 individus (sex ratio de 1,6).

Concernant les mesures de scintigraphie osseuse tardive, 3 heures après injection, la population mesurée est plus importante. Elle est composée de 43 individus (sex ratio de 1,3). La différence entre les mesures faites à 1 mètre et celles faites à 0,5 mètre est significative $(p=0,0043)$. Il est également à noter que la différence entre les deux mesures faites à 1 mètre sur les mêmes patients est significative $(\mathrm{p}=0,017)$ et celles faites à 0,5 mètre aussi $(\mathrm{p}=0,012)$.

\subsection{Résultats de la TEP}

En ce qui concerne la TEP, nous avons fait notre analyse sur une population de 53 individus (sex ratio de 0,56). Ce qui permet une statistique plus stable que dans la plupart des autres cas. Les tests pratiqués à partir des mesures de débit de dose de la population sont donc paramétriques.

\subsection{Résultats de la fraction d'éjection du ventricule gauche}

Pour ce qui est des mesures faites sur des patients sortant d'un examen de leur fraction d'éjection du ventricule gauche, la population est de 8 individus (sex ratio de 3). Celle-ci présentant des problèmes cardiaque est majoritairement masculine. Cela peut s'expliquer par le fait que la grande majorité des hommes ont fumé dans leur jeunesse, ce qui n'est pas forcément le cas pour les femmes d'alors.

\subsection{Résultats pour la scintigraphie cardiaque}

Tous les patients passant une scintigraphie cardiaque ont été mesurés deux fois, une fois après chaque examen, 20 minutes après chaque injection. Une distinction par rapport au nombre d'injection reçut a donc été faite. La population comprend 30 patients (sex ratio de 0,36 ). Après la deuxième injection, la mesure a été faite 2 heures plus tard sur la même population. Si l'on compare les mesures faites à 1 mètre à la première injection et les mesures faites à 1 mètre à la deuxième injection il y a une différence significative entre les deux $(p=0,017)$, également pour les mesures faites à 0,5 mètre $(\mathrm{p}=0,012)$. Nous avons pu mesurer 3 patients passant une scintigraphie myocardique avec du Sestamaibi marqué au Technétium 
TABLEAU II

Doses émises par les patients.

Doses emitted by patients.

\begin{tabular}{|c|c|c|}
\hline TEP & & \\
\hline Activité moyenne injectée (MBq) & $260,4(+/-60,0)$ & \\
\hline Temps moyen entre l'injection et la mesure (h) & $1,89(+/-0,52)$ & \\
\hline Dose moyenne à 1 mètre $(\mu \mathrm{Sv} / \mathrm{h})$ & $10,8(+/-3,5)$ & \\
\hline Dose moyenne à 0,5 mètre $(\mu \mathrm{Sv} / \mathrm{h})$ & $25,0(+/-9,5)$ & \\
\hline Scintigraphie osseuse & Précoce & Tardive \\
\hline Activité moyenne injectée (MBq) & $494,6(+/-113,2)$ & \\
\hline Temps moyen entre l'injection et la mesure (h) & $0,32(+/-0,23)$ & $3,54(+/-0,84)$ \\
\hline Dose moyenne à 1 mètre $(\mu \mathrm{Sv} / \mathrm{h})$ & $7,7(+/-2,7)$ & $4,1(+/-2,0)$ \\
\hline Dose moyenne à 0,5 mètre $(\mu \mathrm{S} v / \mathrm{h})$ & $19,6(+/-9,4)$ & $9,4(+/-5,4)$ \\
\hline Fraction d'éjection du ventricule gauche & & \\
\hline Activité moyenne injectée (MBq) & $860,9(+/-47,3)$ & \\
\hline Temps moyen entre l'injection et la mesure (h) & $0,4(+/-0,14)$ & \\
\hline Dose moyenne à 1 mètre $(\mu \mathrm{Sv} / \mathrm{h})$ & $11,4(+/-3,6)$ & \\
\hline Dose moyenne à 0,5 mètre $(\mu \mathrm{S} v / \mathrm{h})$ & $34,5(+/-8,0)$ & \\
\hline Scintigraphie cardiaque (Thallium) & $1^{\text {ère }}$ injection & $2^{\mathrm{e}}$ injection \\
\hline Activité moyenne injectée (MBq) & $96,0(+/-12,0)$ & $44,4(+/-17,3)$ \\
\hline Temps moyen entre l'injection et la mesure (h) & $0,41(+/-0,22)$ & $1,92(+/-0,7)$ \\
\hline Dose moyenne à 1 mètre $(\mu \mathrm{Sv} / \mathrm{h})$ & $1,2(+/-0,6)$ & $1,3(+/-0,4)$ \\
\hline Dose moyenne à 0,5 mètre $(\mu \mathrm{Sv} / \mathrm{h})$ & $2,3(+/-0,6)$ & $3,1(+/-0,6)$ \\
\hline Scintigraphie cardiaque (Technétium) & $1^{\text {ère }}$ injection & $2^{\mathrm{e}}$ injection \\
\hline Activité moyenne injectée (MBq) & $376,6(+/-41,9)$ & $805,5(+/-91,1)$ \\
\hline Temps moyen entre l'injection et la mesure (h) & $0,65(+/-0,05)$ & $0,6(+/-0,26)$ \\
\hline Dose moyenne à 1 mètre $(\mu \mathrm{Sv} / \mathrm{h})$ & $3,7(+/-0,9)$ & $12,5(+/-2,9)$ \\
\hline Dose moyenne à 0,5 mètre $(\mu \mathrm{Sv} / \mathrm{h})$ & $10,9(+/-2,6)$ & $24,7(+/-12,3)$ \\
\hline Ingestion d'Iode 131 & & \\
\hline Activité moyenne ingérée (MBq) & $397,7(+/-170,4)$ & \\
\hline Dose moyenne à 1 mètre $(\mu \mathrm{Sv} / \mathrm{h})$ & $13,9(+/-5,9)$ & \\
\hline Dose moyenne à 0,5 mètre $(\mu \mathrm{Sv} / \mathrm{h})$ & $39,5(+/-24,2)$ & \\
\hline
\end{tabular}


$99 \mathrm{~m}$ comme radiotraceur. La différence entre les mesures suivant la distance de prise de mesure n'est pas significative $(p=0,11)$. La différence entre les données faites à seconde injection à un mètre et à 0,5 mètre n'est pas significative $(\mathrm{p}=$ $0,18)$. Celle entre les deux mesures faites à 1 mètre la différence n'est pas significative $(\mathrm{p}=0,18)$ et à 0,5 mètre elle ne l'est pas non plus $(\mathrm{p}=0,18)$.

\subsection{Comparaison des périodes radioactives pour l'iode et le technétium}

Le tableau IIIa montre que la durée totale d'émission du patient pour les deux premiers isotopes est courte, moins d'une journée pour le Fluor et moins de deux pour le Technétium. C'est la raison pour laquelle les activités injectées sont importantes. D'après les tableaux IIIa et IIIb, la comparaison de la durée d'émission totale théorique et la durée d'émission totale du cas « réel » montre que la durée totale d'émission est plus courte dans le cas réel que celle du cas théorique pour l'Iode et le Technétium. Également pour la dose totale émise, elle est inférieure dans le cas « réel » par rapport au cas avec la période physique.

\section{Discussion}

Un article sur l'entourage des patients en Médecine nucléaire a déjà été écrit en 2005 où était présenté la diminution de la dose prise au contact, à 1 mètre et à 50 centimètres sur plusieurs jours et sur plusieurs examens. Les résultats de cet article montrent des résultats comparables dans le cas de la mesure faite le jour même. Pour ce qui est des 10 minutes d'exposition du public donné à titre indicatif, il est évident que cela constitue une moyenne. La dose ne sera pas la même si le public est exposé dès la sortie du patient ou quelques temps après.

\subsection{Remarques générales}

En ce qui concerne notre article, le grand écart-type entre les mesures observées sur la quasi-totalité des examens est dû au fait qu'il est demandé aux patients de bien s'hydrater et de passer aux toilettes avant l'examen, ce qui est fait dans la plupart des cas mais pas systématiquement. Cette constatation est la cause des irrégularités de mesures, elle nous est apparue une fois les mesures terminées. Ceci explique également que l'erreur soit plus grande à proximité du patient. De plus, pour certains examens, nous n'avons pas pu faire suffisamment de mesures pour avoir une statistique sans trop d'erreurs. L'écart entre les valeurs à 0,5 mètre est d'autant plus important à cause de cette irrégularité.

Les mesures ont été faites en supposant le rayonnement émis par le patient isotrope. Au cours de ces mesures, un seul patient présenta un débit de dose supérieur à $20 \mu \mathrm{Sv} / \mathrm{h}$. Ce patient, qui venait d'avaler une gélule d'Iode, reçut la 
TABLEAU IIIa

Débit de dose à l'origine et temps d'émission. Dose rate $(a t \mathbf{t}=0)$ and emission time.

\begin{tabular}{|c|c|c|c|c|c|}
\hline \multicolumn{6}{|l|}{ Fluor 18} \\
\hline Période physique (h) & 1,83 & & & & \\
\hline Constante radioactive $\left(\mathrm{h}^{-1}\right)$ & 0,38 & & & & \\
\hline \multirow[t]{2}{*}{ Débit de dose à l'origine ( $\mu \mathrm{Sv} / \mathrm{h})$} & $1 \mathrm{~m}$ & $0,5 \mathrm{~m}$ & & & \\
\hline & 15 & 34,8 & & & \\
\hline Durée d'émission (h) & 13,2 & 15,4 & & & \\
\hline Dose totale émise $(\mu \mathrm{Sv})$ & 28,2 & 65,6 & & & \\
\hline Dose reçue par le public en $10 \mathrm{~min}(\mu \mathrm{Sv})$ & 0,36 & 0,71 & & & \\
\hline \multicolumn{6}{|l|}{ Technétium 99m } \\
\hline \multicolumn{6}{|l|}{ Résultat des régressions par patient } \\
\hline \multirow[t]{10}{*}{ Débit de dose à l'origine $(\mu \mathrm{Sv} / \mathrm{h})$} & $1 \mathrm{~m}$ & $0,5 \mathrm{~m}$ & Période eff & $1 \mathrm{~m}$ & $0,5 \mathrm{~m}$ \\
\hline & 4,42 & 16,2 & & 5,35 & 3,09 \\
\hline & 5,92 & 11,8 & & 2,77 & 4,1 \\
\hline & 2,3 & 8,27 & & 2,47 & 3,4 \\
\hline & 9,58 & 21,3 & & 2,94 & 3,12 \\
\hline & 8,3 & 20 & & 6,9 & 12,3 \\
\hline & 4,4 & 16,3 & & 5,3 & 2,63 \\
\hline & 8,8 & 24,8 & & 4,1 & 5,2 \\
\hline & 9,3 & 24,9 & & 2,4 & 2,8 \\
\hline & 7,4 & 21,4 & & 3,34 & 3,18 \\
\hline \multirow[t]{2}{*}{ Moyenne } & $\begin{array}{c}6,71 \\
(+/-2,57)\end{array}$ & $\begin{array}{c}17,22 \\
(+/-6,98)\end{array}$ & & $\begin{array}{c}3,95 \\
(+/-1,6)\end{array}$ & $\begin{array}{c}4,42 \\
(+/-3,06)\end{array}$ \\
\hline & & & Moyenne & $\begin{array}{c}4,18 \\
(+/-4,23)\end{array}$ & \\
\hline Durée d'émission (h) & 17,7 & 21,5 & & & \\
\hline Dose totale émise $(\mu \mathrm{Sv})$ & 27,7 & 71,55 & & & \\
\hline Dose reçue par le public en $10 \mathrm{~min}(\mu \mathrm{Sv})$ & 0,26 & 0,55 & & & \\
\hline \multicolumn{6}{|l|}{ Cas théorique } \\
\hline Période physique (h) & 6,02 & & & & \\
\hline Constante radioactive $\left(\mathrm{h}^{-1}\right)$ & 0,12 & & & & \\
\hline Débit de dose à l'origine ( $\mu \mathrm{Sv} / \mathrm{h})$ & 6,7 & 18,3 & & & \\
\hline Durée d'émission (h) & 35 & 43,4 & & & \\
\hline Dose totale émise $(\mu \mathrm{Sv})$ & 55 & 151,7 & & & \\
\hline Dose reçue par le public en $10 \min (\mu \mathrm{Sv})$ & 0,27 & 0,59 & & & \\
\hline
\end{tabular}

dose maximale prescriptible dans le service en une fois. Il est à rappeler ici que l'ingestion d'Iode est réalisée en vue d'un traitement et non d'un examen d'imagerie, bien que cela soit possible. 
TABLEAU IIIb

Débit de dose à l'origine et durée d'émission.

Dose rate $($ at $t=0)$ and emission time.

\begin{tabular}{|c|c|c|}
\hline Thallium 201 & & \\
\hline Période physique (h) & 73 & \\
\hline Constante radioactive (h-1) & 0,0094 & \\
\hline Ordonnée à l'origine ( $\mu \mathrm{Sv} / \mathrm{h})$ & à $1 \mathrm{~m}$ & à $0,5 \mathrm{~m}$ \\
\hline & 1,35 & 3,2 \\
\hline Durée d'émission (h) & 276,8 & 368,7 \\
\hline Dose totale émise $(\mu \mathrm{Sv})$ & 132,4 & 329,8 \\
\hline Dose reçue par le public en $10 \mathrm{~min}(\mu \mathrm{Sv})$ & 0,08 & 0,15 \\
\hline Iode 131 & & \\
\hline Cas théorique & 192 & \\
\hline Période physique (h) & & \\
\hline Constante radioactive $\left(\mathrm{h}^{-1}\right)$ & 0,0036 & \\
\hline Ordonnée à l'origine $(\mu \mathrm{Sv} / \mathrm{h})$ & à $1 \mathrm{~m}$ & à $0,5 \mathrm{~m}$ \\
\hline & 13,9 & 39,5 \\
\hline Durée d'émission (h) & 1370,7 & 1660,8 \\
\hline Dose totale émise $(\mu \mathrm{Sv})$ & 3833,3 & 10944,4 \\
\hline Dose reçue par le public en $10 \mathrm{~min}(\mu \mathrm{Sv})$ & 0,47 & 1,1 \\
\hline Cas « réel » & & \\
\hline Période effective (h) & 168 & \\
\hline Constante radioactive (h-1) & 0,0041 & \\
\hline Ordonnée à l'origine ( $\mu \mathrm{Sv} / \mathrm{h})$ & à $1 \mathrm{~m}$ & à $0,5 \mathrm{~m}$ \\
\hline & 13,9 & 39,5 \\
\hline Durée d'émission (h) & 1203,5 & 1458,3 \\
\hline Dose totale émise $(\mu \mathrm{Sv})$ & 3365,8 & 9609,8 \\
\hline Dose reçue par le public en $10 \mathrm{~min}(\mu \mathrm{Sv})$ & 0,47 & 1,1 \\
\hline
\end{tabular}

Les moyennes d'âge correspondent à des personnes souffrant de maladies en relation avec l'augmentation de l'âge. C'est ce type de maladie qui est le plus souvent observé en imagerie nucléaire. Ce n'est pas un échantillon représentatif de la population générale mais de celle qui vient dans ce service.

\section{2. À propos de la scintigraphie osseuse}

Le minimum d'activité injectée de $550 \mathrm{MBq}$ en dessous duquel il devient difficile d'obtenir une bonne qualité d'image ne permet pas de montrer la relation de proportionnalité entre activité injectée et poids. La corrélation entre poids et activité injectée se serait vue si l'échantillon avait eu un poids moyen égal ou 
supérieur à $74 \mathrm{~kg}$. La grande majorité des personnes mesurées présentant un poids inférieur à $74 \mathrm{~kg}$, la dose injectée fut quasiment la même pour toutes les personnes ayant réalisé cet examen d'imagerie. Si nous avions pu prolonger la période de mesure, nous aurions pu obtenir cette corrélation.

\section{3. À propos de la scintigraphie cardiaque au Thallium}

La corrélation entre le temps et la dose émise par le patient n'a pas pu être mise en évidence car le faible écart entre les temps où la mesure était faite est négligeable devant la période du radionucléide. Selon le code de l'environnement, si une personne ayant reçu une injection de produit radioactif présente un débit de dose inférieur à $2,5 \mu \mathrm{Sv} / \mathrm{h}$, elle peut ne pas être soumise aux contrôles de radioprotection et donc ne pas observer les règles de sécurité comme l'éloignement durant la période de radioactivité. Cela pourrait être le cas pour un patient sortant de cet examen.

\section{4. À propos de la scintigraphie osseuse}

Les patients qui ont été mesurés deux fois après avoir passé une scintigraphie osseuse ont bien permis de voir que la radioactivité diminuait au cours du temps, d'une part physiquement car la période du technétium est de 6 heures mais aussi grâce à l'élimination biologique d'une partie de la radioactivité non fixée dans l'organisme.

\section{5. À propos de la scintigraphie cardiaque au Technétium}

Pour les patients ayant eu une scintigraphie myocardique au Technétium le nombre insuffisant de mesures ne permet pas de conclure à une différence significative par rapport à la distance. Cependant, il subsiste une différence de débit de dose suivant le choix de l'isotope. En effet, le débit de dose émis par le patient sera plus élevé à la sortie du service s'il a eu un examen au Technétium.

\subsection{Remarques sur les calculs}

L'ordonnée à l'origine a été obtenue par résolution de l'équation de décroissance radioactive à l'aide de la moyenne des valeurs mesurées et la moyenne des temps entre injection et mesure. Dans le cas du Thallium, seul le dernier point a été pris en compte. Cela a simplement conduit à une majoration des résultats pour donner un ordre d'idée dans « le pire des cas ».

La durée d'émission et la quantité de rayonnement émis pour le public se révèlent être peu différents car même si la différence de période est grande, le 
temps d'exposition potentiel reste faible comparé aux périodes. En revanche, les quantités totales émises différent bien suivant la période choisie. Ce qui montre bien que le choix de la période physique constitue une majoration des valeurs.

\section{Conclusion}

Sur les 145 patients mesurés, seul un a présenté une valeur au-delà de la limite de $20 \mu \mathrm{Sv} / \mathrm{h}$. La période physique étant relativement longue, ce patient devra donc se tenir à l'écart de la population à risque pendant la semaine qui suit, c'est-à-dire à plusieurs mètres. Cette population est constituée des femmes enceintes et des enfants en bas âge.

À la question : Si nous restons à côté du patient pendant tout ou une partie du temps où il est radioactif, recevons-nous une dose égale ou supérieure à $1 \mathrm{mSv}$ ?

Les débits de dose trouvés sont trop faibles pour atteindre ou dépasser la limite de $1 \mathrm{mSv} / \mathrm{an}$. À l'exception de l'iode 131, aucun isotope ne délivre une dose totale aussi élevée. Autrement dit, aucun patient ayant bénéficié d'un examen d'imagerie ne délivre une dose environnementale supérieure à $1 \mathrm{mSv}$. Une personne non aidante devrait rester en moyenne 14 jours sur 51 jours à 1 mètre et 6 jours sur 61,5 jours à 0,5 mètre aux cotés d'un patient ayant avalé une pastille d'iode pour avoir $1 \mathrm{mSv}$. Cela correspond à rester 6,5 heures par jour à 1 mètre du patient et 2,3 heures par jour à 0,5 mètre, tous les jours sur les mêmes durées. Sachant qu'il s'agit d'un traitement, les quantités sont forcément plus importantes que pour une simple image, il est donc normal, pour des raisons thérapeutiques, que les patients reçoivent une activité importante et émettent une quantité plus grande de rayonnements. Les personnes ayant reçu un traitement à l'iode 131 ont des consignes de radioprotection claires qu'on leur demande de respecter une fois sortis du service.

Étant donné que la gélule d'Iode est avalée avec de l'eau, le patient peut devenir une source de contamination par rejet de postillons. Il pourrait être intéressant de voir à quel point cela doit être pris en compte.

\section{RÉFÉRENCES}

Articles R.231-73 à R.231-116 du Code du travail.

Articles L.1333-1 à L.1333-18 et R.1333-1 à R.1333-92 du Code de la santé publique.

Catalogue Radioprotection (2002) pp IV-1. Eurisys Mesures.

Code de l'environnement, Article L542-1-1.

Delacroix D. et al. (2004) Radionucléides et Radioprotection. EDP Sciences.

http://www.irsn.fr/FR/professionnels_sante/radiopro_travailleurs/Documents/medecine_nucleaire/iod e 131.pdf (à la date du 07/06/2011).

http://www.mesure-radioactivite.fr/public/spip.php?rubrique69 (à la date du 07/06/2011).

Gomez-Palacios M. et al. (2005) Radiation Doses in the Surrounding of Patients Undergoing Nuclear Medecine Diagnostic Studies, 89, pp. S27-S34. 